Pure and Applied Mathematics Quarterly

Volume 6, Number 4

(Special Issue: In honor of

Joseph J. Kohn, Part 2 of 2$)$

$1145-1167,2010$

\title{
Number Variance of Random Zeros on Complex Manifolds, II: Smooth Statistics
}

\author{
Bernard Shiffman and Steve Zelditch \\ To Joseph J. Kohn on the occasion of his 75th birthday
}

\begin{abstract}
We consider the zero sets $Z_{N}$ of systems of $m$ random polynomials of degree $N$ in $m$ complex variables, and we give asymptotic formulas for the random variables given by summing a smooth test function over $Z_{N}$. Our asymptotic formulas show that the variances for these smooth statistics have the growth $N^{m-2}$. We also prove analogues for the integrals of smooth test forms over the subvarieties defined by $k<m$ random polynomials. Such linear statistics of random zero sets are smooth analogues of the random variables given by counting the number of zeros in an open set, which we proved elsewhere to have variances of order $N^{m-1 / 2}$. We use the variance asymptotics and off-diagonal estimates of Szegö kernels to extend the central limit theorem of Sodin-Tsirelson to the case of smooth linear statistics for zero sets of codimension one in any dimension $m$.
\end{abstract}

Keywords: Random holomorphic sections, zeros of random polynomials, positive line bundle, Kähler manifold, Szegő kernel

\section{INTRODUCTION}

This article is concerned with zero sets of systems of Gaussian random polynomials (or more generally, of sections of a positive holomorphic line bundle over

Received November 12, 2007.

Research of the first author partially supported by NSF grant DMS-0600982; research of the second author partially supported by NSF grant DMS-0603850. 
a compact Kähler manifold $M)$ as the degree $N \rightarrow \infty$. One of the most fundamental statistical quantities is the number $\mathcal{N}_{N}^{U}\left(p_{1}^{N}, \ldots, p_{m}^{N}\right)$ of zeros in a bounded open set $U \subset \mathbb{C}^{m}$ of a system $\left\{p_{1}^{N}, \ldots, p_{m}^{N}\right\}$ of $m$ independent Gaussian random polynomials. The expected value of this random variable was shown in [SZ1] to be the integral of the Fubini-Study volume form over $U$ (times a universal constant). In a recent article [SZ4], we gave an asymptotic formula for the variance of this random variable. We also give analogous results for the volume of the simultaneous zero set of $k<m$ polynomials or sections. In this article we apply the methods of [SZ4] to the analogous 'smooth linear statistics', i.e. the sum (or integral) of a smooth test function over the zeros of a system of random polynomials. Such smooth linear statistics arise as smooth approximations for discontinuous random variables such as $\mathcal{N}_{N}^{U}$ and also arise in a number of other problems (see the discussion in $\S 5$ ). Our main results give asymptotic formulas for the variance of these smooth linear statistics. (Mean value asymptotics for these random variables were given in [SZ1].) As may be expected, the variances are of much lower order in the degree $N$ than in the non-smooth case. Further, we prove that in the codimension one case, the smooth linear statistics are asymptotically normal, extending a result of Sodin-Tsirelson [ST].

To state our results precisely, we need some notation and background. We let $(L, h) \rightarrow M$ be a positively curved Hermitian holomorphic line bundle over a compact complex manifold $M$ of dimension $m$, and we give $H^{0}\left(M, L^{N}\right)$ the Hermitian Gaussian measure induced by $h$ and the Kähler form $\omega=\frac{i}{2} \Theta_{h}$ (see Definition 2.1). For $m$ independent random sections $s_{j}^{N} \in H^{0}\left(M, L^{N}\right), 1 \leq j \leq$ $m$, the number of simultaneous zeros of the sections in a smooth domain $U \subset M$ is given by

$$
\mathcal{N}_{N}^{U}\left(s_{1}^{N}, \ldots, s_{m}^{N}\right):=\#\left\{z \in U: s_{1}^{N}(z)=\cdots=s_{m}^{N}(z)=0\right\} .
$$

In [SZ1], we proved that the expected value $\mathbf{E}\left(\mathcal{N}_{N}^{U}\right)$ of the random variable $\mathcal{N}_{N}^{U}$ has the asymptotics

$$
\mathbf{E}\left(\mathcal{N}_{N}^{U}\right)=\frac{N^{m}}{\pi^{m}} \int_{U} \omega^{m}+O\left(N^{m-1}\right)
$$

and in [SZ4], the variance of the random variable is shown to have the asymptotics,

$$
\operatorname{Var}\left(\mathcal{N}_{N}^{U}\right)=N^{m-1 / 2}\left[\nu_{m m} \operatorname{Vol}_{2 m-1}(\partial U)+O\left(N^{-\frac{1}{2}+\varepsilon}\right)\right]
$$


where $\nu_{m m}$ is a universal positive constant ([SZ4, Theorem 1.1]). Analogous results are proved for the volumes of zero sets of $k \leq m-1$ independent random sections $s_{j}^{N} \in H^{0}\left(M, L^{N}\right), 1 \leq j \leq k$ : in this case,

$$
\operatorname{Var}\left(\operatorname{Vol}_{2 m-2 k}\left[Z_{s_{1}^{N}, \ldots, s_{k}^{N}} \cap U\right]\right)=N^{2 k-m-1 / 2}\left[\nu_{m k} \operatorname{Vol}_{2 m-1}(\partial U)+O\left(N^{-\frac{1}{2}+\varepsilon}\right)\right],
$$

where $\nu_{m k}$ is a universal positive constant ([SZ4, Theorem 1.4]); in particular, $\nu_{m 1}=\frac{\pi^{m-5 / 2}}{8} \zeta\left(m+\frac{1}{2}\right)$. More generally, the domain could be piecewise smooth without cusps.

In this article, we are interested in the smooth analogue of $\mathcal{N}_{N}^{U}$ where we integrate a smooth test function rather than the characteristic function of a smooth domain over the zero set. Given a test function $\varphi \in \mathcal{D}(M)$, we consider the random variable

$$
\left(Z_{s_{1}^{N}, \ldots, s_{m}^{N}}, \varphi\right)=\sum_{s_{1}^{N}(z)=\cdots=s_{m}^{N}(z)=0} \varphi(z) .
$$

When the system is not full, we define

$$
\left(Z_{s_{1}^{N}, \ldots, s_{k}^{N}}, \varphi\right)=\int_{s_{1}^{N}(z)=\cdots=s_{k}^{N}(z)=0} \varphi(z), \quad \varphi \in \mathcal{D}^{m-k, m-k}(M) .
$$

The expected value of $\left(Z_{s_{1}^{N}, \ldots, s_{m}^{N}}, \varphi\right)$ is given by (see (14))

$$
\mathbf{E}\left(Z_{s_{1}^{N}, \ldots, s_{k}^{N}}, \varphi\right) \approx N^{k} \pi^{-k} \int_{M} \omega^{k} \wedge \varphi .
$$

The main result of this article is an asymptotic formula for the variance:

TheOREm 1.1. Let $(L, h)$ be a positive Hermitian holomorphic line bundle over a compact Kähler manifold $(M, \omega)$, where $\omega=\frac{i}{2} \Theta_{h}$, and let $1 \leq k \leq m=$ $\operatorname{dim} M$. We give $H^{0}\left(M, L^{N}\right)$ the Hermitian Gaussian measure induced by $h, \omega$ (see Definition 2.1).

Let $\varphi$ be a real $(m-k, m-k)$-form on $M$ with $\mathcal{C}^{3}$ coefficients. Then for independent random sections $s_{1}^{N}, \ldots, s_{k}^{N} \in H^{0}\left(M, L^{N}\right)$, we have

$$
\operatorname{Var}\left(Z_{s_{1}^{N}, \ldots, s_{k}^{N}}, \varphi\right)=N^{2 k-m-2}\left[\int_{M} B_{m k}(\partial \bar{\partial} \varphi, \partial \bar{\partial} \varphi) \Omega_{M}+O\left(N^{-\frac{1}{2}+\varepsilon}\right)\right],
$$

where $\Omega_{M}$ is the volume form on $M$, and $B_{m k}$ is a universal Hermitian form on the bundle $T^{* m-k+1, m-k+1}(M)$. 
When $k=1$, we have $B_{m 1}\left(f \Omega_{M}, f \Omega_{M}\right)=\frac{\pi^{m-2} \zeta(m+2)}{4}|f|^{2}$, and hence

$$
\operatorname{Var}\left(Z_{s^{N}}, \varphi\right)=N^{-m}\left[\frac{\pi^{m-2} \zeta(m+2)}{4}\|\partial \bar{\partial} \varphi\|_{L^{2}}^{2}+O\left(N^{-\frac{1}{2}+\varepsilon}\right)\right] .
$$

In particular, for the complex curve case $m=1$, we note that $|\partial \bar{\partial} \varphi|=\frac{1}{2}|\Delta \varphi|$, and thus

$$
\operatorname{Var}\left(Z_{s^{N}}, \varphi\right)=N^{-1}\left[\frac{\zeta(3)}{16 \pi}\|\Delta \varphi\|_{2}^{2}+O\left(N^{-\frac{1}{2}+\varepsilon}\right)\right] .
$$

The leading term in (5) was obtained by Sodin and Tsirelson [ST] for the case of random polynomials $s^{N} \in H^{0}\left(\mathbb{C P}^{1}, \mathcal{O}(N)\right)$ as well as for random holomorphic functions on $\mathbb{C}$ and on the disk. (The constant $\frac{\zeta(3)}{16 \pi}$ was given in a private communication from M. Sodin.)

Here we say that $B_{m k}$ is universal if there exists a Hermitian inner product $B_{m k}^{0}$ on $T_{0}^{* m-k+1, m-k+1}\left(\mathbb{C}^{m}\right)$, independent of $M$ and $L$, such that for all $w \in M$ and all unitary transformations $\tau: T_{0}^{*}\left(\mathbb{C}^{m}\right) \rightarrow T_{w}^{*}(M)$, we have $B_{m k}(w)=\tau_{*} B_{m k}^{0}$. The global inner product $(\varphi, \psi)=\int_{M} B_{m k}(\partial \bar{\partial} \varphi, \partial \bar{\partial} \psi) \Omega_{M}$ is certainly positive semi-definite on $\mathcal{D}^{m-k, m-k}(M)$, since the variance is nonnegative. We believe that, in fact, $B_{m k}^{0}$ is positive definite on $T_{0}^{* m-k+1, m-k+1}\left(\mathbb{C}^{m}\right)$. This follows for $k=1$ from the above formula for $B_{m 1}$; one should be able to verify positivity for $k>1$ by using the expansion (27) in the proof of Theorem 1.1 to compute a precise formula for $B_{m k}^{0}$.

Thus the variance of the 'smooth statistic' $\left(Z_{s_{1}^{N}, \ldots, s_{k}^{N}}, \varphi\right)$ is of lower order than the variance of the number and volume statistics given by (2)-(3), as expected. In view of (4), it is also self-averaging in the sense that its fluctuations are of smaller order than its typical values.

An application of our methods is an extension of the Sodin-Tsirelson [ST] central limit theorem for smooth statistics to general one-dimensional ensembles and to codimension one zero sets in higher dimensions:

TheOREM 1.2. Let $(L, h) \rightarrow(M, \omega)$ be as in Theorem 1.1 and give $H^{0}\left(M, L^{N}\right)$ the Hermitian Gaussian measure induced by $h, \omega$. Let $\varphi$ be a real $(m-1, m-1)$ form on $M$ with $\mathcal{C}^{3}$ coefficients, such that $\partial \bar{\partial} \varphi \not \equiv 0$. Then for random sections $s^{N}$ in $H^{0}\left(M, L^{N}\right)$, the distributions of the random variables

$$
\frac{\left(Z_{s^{N}}, \varphi\right)-\mathbf{E}\left(Z_{s^{N}}, \varphi\right)}{\sqrt{\operatorname{Var}\left(Z_{s^{N}}, \varphi\right)}}
$$

converge weakly to the standard Gaussian distribution $\mathcal{N}(0,1)$ as $N \rightarrow \infty$. 
Sodin and Tsirelson [ST] obtained the asymptotics of Theorem 1.2 for random functions on $\mathbb{C}, \mathbb{C P}^{1}$, and the disk. The proof of Theorem 1.2 is a relatively straightforward application of the fundamental Szegö kernel asymptotics underlying Theorem 1.1 to the argument in $[\mathrm{ST}]$. (One easily sees that the random variable $\left(Z_{s^{N}}, \varphi\right)$ is constant for all $N$ if $\partial \bar{\partial} \varphi \equiv 0$.)

Substituting the values of the expectation and variance of $\left(Z_{s^{N}}, \varphi\right)$ from (4) and Theorem 1.1, respectively, we have:

Corollary 1.3. With the same notation and hypotheses as in Theorem 1.2, the distributions of the random variables $N^{m / 2}\left(Z_{s^{N}}-\frac{N}{\pi} \omega, \varphi\right)$ converge weakly to $\mathcal{N}\left(0, \sqrt{\kappa_{m}}\|\partial \bar{\partial} \varphi\|_{2}\right)$ as $N \rightarrow \infty$, where $\kappa_{m}=\frac{\pi^{m-2} \zeta(m+2)}{4}$.

Here, $\mathcal{N}(0, \sigma)$ denotes the (real) Gaussian distribution of mean zero and variance $\sigma^{2}$.

We now summarize the key ideas in the proofs in [SZ4] and in this paper. The variance in Theorem 1.1, as well as the number and volume variances in [SZ4], can be expressed in terms of the variance currents $\operatorname{Var}\left(Z_{s_{1}^{N}, \ldots, s_{k}^{N}}\right)$ of the random currents $Z_{s_{1}^{N}, \ldots, s_{k}^{N}}$. In joint work with P. Bleher in 2000 [BSZ1], we introduced a bipotential $Q_{N}$ for the 'pair correlation function' $K_{21}^{N}$ of the volume density of zeros of random sections in $H^{0}\left(M, L^{N}\right)$; this bipotential satisfies

$$
\Delta_{z} \Delta_{w} Q_{N}(z, w)=K_{21}^{N}(z, w) .
$$

The bipotential $Q_{N}$ is a universal function of the normalized Szegő kernel (see (16) and (22)). Sodin and Tsirelson [ST] obtained a variance formula as well as a 'central limit theorem' for zeros of certain model one-dimensional random holomorphic functions by implicitly using this bipotential.

In [SZ4], we showed that $Q_{N}$ is actually a 'pluri-bipotential' for the codimensionone variance current; i.e.,

$$
(i \partial \bar{\partial})_{z}(i \partial \bar{\partial})_{w} Q_{N}(z, w)=\operatorname{Var}\left(Z_{s^{N}}\right) .
$$

We further found a formula (Theorem 3.1) for the higher codimension variance current $\operatorname{Var}\left(Z_{s_{1}^{N}, \ldots, s_{k}^{N}}\right)$ in terms of $Q_{N}$ and its derivatives of order $\leq 4$. We then applied the off-diagonal asymptotics of the Szegö kernel $\Pi_{N}(z, w)$ in [SZ2] to obtain asymptotics of the bipotential $Q_{N}(z, w)$ and then of the number variance (2) as well as the volume variance (3). 
In this paper, we begin by reviewing basic facts about the Szegö kernel and summarizing the asymptotics from [SZ4] of the bipotential $Q_{N}$ as $N \rightarrow \infty$ as well as $d(z, w) \rightarrow 0$. To illustrate our ideas, we apply these asymptotics to (6) to derive the codimension one formula (i.e., the case $k=1$ ) of Theorem 1.1. We then prove in $\S 3.2$ a slight modification (Corollary 3.3) of the formula for the higher codimension variance, which we use in $\S 3.3$ to prove Theorem 1.1. In $\S 4$ we apply our Szegő kernel asymptotics to prove Theorem 1.2. Finally, we formulate some related open problems in $\S 5$.

\section{BACKGROUND}

In this section we summarize results from [SZ4] used in this paper.

We let $(L, h)$ be a Hermitian holomorphic line bundle over a compact Kähler manifold $M$. We consider a local holomorphic frame $e_{L}$ over a trivializing chart $U$. If $s=f e_{L}$ is a section of $L$ over $U$, its Hermitian norm is given by $\|s(z)\|_{h}=$ $a(z)^{-\frac{1}{2}}|f(z)|$ where

$$
a(z)=\left\|e_{L}(z)\right\|_{h}^{-2} .
$$

The curvature form of $(L, h)$ is given locally by

$$
\Theta_{h}=\partial \bar{\partial} \log a,
$$

and the Chern form $c_{1}(L, h)$ is given by

$$
c_{1}(L, h)=\frac{\sqrt{-1}}{2 \pi} \Theta_{h}=\frac{\sqrt{-1}}{2 \pi} \partial \bar{\partial} \log a .
$$

The current of integration $Z_{s}$ over the zeros of a section $s \in H^{0}(M, L)$ is then given by the Poincaré-Lelong formula,

$$
Z_{s}=\frac{\sqrt{-1}}{\pi} \partial \bar{\partial} \log |f|=\frac{\sqrt{-1}}{\pi} \partial \bar{\partial} \log \|s\|_{h}+c_{1}(L, h),
$$

where the second equality is a consequence of (7)-(8).

We now assume that the Hermitian metric $h$ has strictly positive curvature and we give $M$ the Kähler form

$$
\omega=\frac{i}{2} \Theta_{h}=\pi c_{1}(L, h) .
$$

Next we describe the natural Gaussian probability measures on the spaces $H^{0}\left(M, L^{N}\right)$ of holomorphic sections of tensor powers $L^{N}=L^{\otimes N}$ of the line bundle $L$ : 
Definition 2.1. Let $(L, h) \rightarrow(M, \omega)$ be as above, and let $h^{N}$ denote the Hermitian metric on $L^{N}$ induced by $h$. We give $H^{0}\left(M, L^{N}\right)$ the inner product induced by the Kähler form $\omega$ and the Hermitian metric $h^{N}$ :

$$
\left\langle s_{1}, \bar{s}_{2}\right\rangle=\int_{M} h^{N}\left(s_{1}, s_{2}\right) \frac{1}{m !} \omega^{m}, \quad s_{1}, s_{2} \in H^{0}\left(M, L^{N}\right) .
$$

The Hermitian Gaussian measure on $H^{0}\left(M, L^{N}\right)$ is the complex Gaussian probability measure $\gamma_{N}$ induced by the inner product (11):

$$
d \gamma_{N}(s)=\frac{1}{\pi^{m}} e^{-|c|^{2}} d c, \quad s=\sum_{j=1}^{d_{N}} c_{j} S_{j}^{N},
$$

where $\left\{S_{1}^{N}, \ldots, S_{d_{N}}^{N}\right\}$ is an orthonormal basis for $H^{0}\left(M, L^{N}\right)$. It is of course independent of the choice of orthonormal basis.

The Gaussian ensembles $\left(H^{0}\left(M, L^{N}\right), \gamma_{N}\right)$ were also studied in [BSZ1, BSZ2, $\mathrm{SZ1}, \mathrm{SZ2}, \mathrm{SZZ}]$; for the case of polynomials in one variable, they become the $\mathrm{SU}(2)$ ensembles in [BBL, Ha, NV, Zh]; for polynomials in $m$ complex variables, they are the $\mathrm{SU}(m+1)$ ensembles (see, e.g., [BSZ2, SZ1]).

We consider the diagonal Szegő kernels

$$
\Pi_{N}(z, z):=\sum_{j=1}^{d_{N}}\left\|S_{j}^{N}(z)\right\|_{h^{N}(z)}^{2},
$$

where the $S_{j}^{N}$ are as in the above definition. It follows from the leading terms of the asymptotic expansion of the diagonal Szegö kernel of [Ca, Ti, Ze] that

$$
\Pi_{N}(z, z)=\frac{1}{\pi^{m}} N^{m}\left(1+O\left(N^{-1}\right)\right) .
$$

The expected value of the zero current of a random holomorphic section in $H^{0}\left(M, L^{N}\right)$ is given by the basic formula

$$
\mathbf{E} Z_{s^{N}}=\frac{i}{2 \pi} \partial \bar{\partial} \log \Pi_{N}(z, z)+\frac{N}{\pi} \omega
$$

and the expected values of simultaneous zero currents are given by

$$
\mathbf{E}\left(Z_{s_{1}^{N}, \ldots, s_{k}^{N}}\right)=\left[\mathbf{E}\left(Z_{s^{N}}\right)\right]^{\wedge k}=\left(\frac{i}{2 \pi} \partial \bar{\partial} \log \Pi_{N}(z, z)+\frac{N}{\pi} \omega\right)^{k}=\frac{N^{k}}{\pi^{k}} \omega^{k}+O\left(N^{k-1}\right),
$$

for $1 \leq k \leq m$ (see [SZ1, SZ4]). The final equality of (14) is a consequence of the asymptotic formula (12). 
For the variance asymptotics, we need the properties of the off-diagonal Szegö kernel:

$$
\left|\Pi_{N}(z, w)\right|:=\left\|\sum_{j=1}^{d_{N}} S_{j}^{N}(z) \otimes \overline{S_{j}^{N}(w)}\right\|_{h^{N}(z) \otimes h^{N}(w)} .
$$

In particular, our variance formulas are expressed in terms of the normalized Szegö kernel

$$
P_{N}(z, w):=\frac{\left|\Pi_{N}(z, w)\right|}{\Pi_{N}(z, z)^{\frac{1}{2}} \Pi_{N}(w, w)^{\frac{1}{2}}} .
$$

In [SZ4], we used the off-diagonal asymptotics for $\Pi_{N}$ from [SZ2] to provide the off-diagonal estimates given below for the normalized Szegö kernel $P_{N}$. As in [BSZ1, SZ1, SZ2, SZ4]), we obtained these asymptotics by identifying the line bundle Szegö kernel $\left|\Pi_{N}(z, w)\right|$ of (15) with the absolute value of a scalar Szegö kernel $\Pi_{N}(x, y)$ on the unit circle bundle $X \subset L^{-1} \rightarrow M$ associated to the Hermitian metric $h$.

Our estimates are of two types: (1) 'near-diagonal' asymptotics (Proposition 2.3) for $P_{N}(z, w)$ where the distance $d(z, w)$ between $z$ and $w$ satisfies an upper bound $d(z, w) \leq b\left(\frac{\log N}{N}\right)^{1 / 2}\left(b \in \mathbb{R}^{+}\right) ;(2)$ 'far-off-diagonal' asymptotics (Proposition 2.2) where $d(z, w) \geq b\left(\frac{\log N}{N}\right)^{1 / 2}$ :

Proposition 2.2. [SZ4, Prop. 2.6] Let $(L, h) \rightarrow(M, \omega)$ be as in Theorem 1.1, and let $P_{N}(z, w)$ be the normalized Szegö kernel for $H^{0}\left(M, L^{N}\right)$ given by (16). For $b>\sqrt{j+2 k}, j, k \geq 0$, we have

$$
\nabla^{j} P_{N}(z, w)=O\left(N^{-k}\right) \quad \text { uniformly for } d(z, w) \geq b \sqrt{\frac{\log N}{N}} .
$$

Here, $\nabla^{j}$ stands for the $j$-th covariant derivative. The normalized Szegö kernel $P_{N}$ also satisfies Gaussian decay estimates valid very close to the diagonal. To give this estimate, we consider a local normal coordinate chart $\rho: U, z_{0} \rightarrow \mathbb{C}^{m}, 0$ centered at a point $z_{0} \in U \subset M$, and we write, by abuse of notation,

$$
P_{N}\left(z_{0}+u, z_{0}+v\right):=P_{N}\left(\rho^{-1}(u), \rho^{-1}(v)\right) .
$$

Proposition 2.3. [SZ4, Prop. 2.7-2.8] Let $P_{N}(z, w)$ be as in Proposition 2.2, and let $z_{0} \in M$. For $\varepsilon, b>0$, there are constants $C_{j}=C_{j}(M, \varepsilon, b), j \geq 2$, 
independent of the point $z_{0}$, such that

$$
P_{N}\left(z_{0}+\frac{u}{\sqrt{N}}, z_{0}+\frac{v}{\sqrt{N}}\right)=e^{-\frac{1}{2}|u-v|^{2}}\left[1+R_{N}(u, v)\right],
$$

where

$$
\begin{gathered}
\left|R_{N}(u, v)\right| \leq \frac{C_{2}}{2}|u-v|^{2} N^{-1 / 2+\varepsilon}, \quad\left|\nabla R_{N}(u)\right| \leq C_{2}|u-v| N^{-1 / 2+\varepsilon}, \\
\left|\nabla^{j} R_{N}(u, v)\right| \leq C_{j} N^{-1 / 2+\varepsilon} \quad j \geq 2,
\end{gathered}
$$

for $|u|+|v|<b \sqrt{\log N}$.

2.1. The pluri-bipotential for the variance. For random codimension $k$ zeros, we have the variance current of $Z_{s_{1}^{N}, \ldots, s_{k}^{N}}$ :

$$
\begin{aligned}
\operatorname{Var}\left(Z_{s_{1}^{N}, \ldots, s_{k}^{N}}\right)=\mathbf{E}\left(Z_{s_{1}^{N}, \ldots, s_{k}^{N}} \otimes Z_{s_{1}^{N}, \ldots, s_{k}^{N}}\right) & \\
& \quad-\mathbf{E}\left(Z_{s_{1}^{N}, \ldots, s_{k}^{N}}\right) \otimes \mathbf{E}\left(Z_{s_{1}^{N}, \ldots, s_{k}^{N}}\right) \in \mathcal{D}^{\prime 2 k, 2 k}(M \times M) .
\end{aligned}
$$

The variance for the 'smooth zero statistics' is given by:

$$
\operatorname{Var}\left(Z_{s_{1}^{N}, \ldots, s_{k}^{N}}, \varphi\right)=\left(\operatorname{Var}\left(Z_{s_{1}^{N}, \ldots, s_{k}^{N}}\right), \varphi \otimes \varphi\right) .
$$

Here we write

$$
R \otimes S=\pi_{1}^{*} R \wedge \pi_{2}^{*} S \in \mathcal{D}^{\prime p+q}(M \times M), \quad \text { for } R \in \mathcal{D}^{\prime p}(M), S \in \mathcal{D}^{\prime q}(M),
$$

where $\pi_{1}, \pi_{2}: M \times M \rightarrow M$ are the projections to the first and second factors, respectively.

For a current $T$ on $M \times M$, we shall write

$$
\partial T=\partial_{1} T+\partial_{2} T, \quad \partial_{1}=\sum d z_{j} \frac{\partial}{\partial z_{j}}, \quad \partial_{2}=\sum d w_{j} \frac{\partial}{\partial w_{j}},
$$

where $z_{1}, \ldots, z_{m}$ are local coordinates on the first factor, and $w_{1}, \ldots, w_{m}$ are local coordinates on the second factor of $M \times M$. In particular, $\partial_{1}(R \otimes S)=(\partial R) \otimes S$ and $\partial_{2}(R \otimes S)=R \otimes(\partial S)$. We similarly write

$$
\bar{\partial} T=\bar{\partial}_{1} T+\bar{\partial}_{2} T
$$

In [SZ4], we constructed a pluri-bipotential for the variance current in codimension one, i.e. a function $Q_{N} \in L^{1}(M \times M)$ such that

$$
\operatorname{Var}\left(Z_{s^{N}}\right)=-\partial_{1} \bar{\partial}_{1} \partial_{2} \bar{\partial}_{2} Q_{N}=(i \partial \bar{\partial})_{z}(i \partial \bar{\partial})_{w} Q_{N}(z, w) .
$$


To describe our pluri-bipotential $Q_{N}(z, w)$, we define the function

$$
\widetilde{G}(t):=-\frac{1}{4 \pi^{2}} \int_{0}^{t^{2}} \frac{\log (1-s)}{s} d s=\frac{1}{4 \pi^{2}} \sum_{n=1}^{\infty} \frac{t^{2 n}}{n^{2}}, \quad 0 \leq t \leq 1 .
$$

Alternatively,

$$
\widetilde{G}\left(e^{-\lambda}\right)=-\frac{1}{2 \pi^{2}} \int_{\lambda}^{\infty} \log \left(1-e^{-2 s}\right) d s, \quad \lambda \geq 0 .
$$

Theorem 2.4. [SZ4, Theorem 3.1] Let $(L, h) \rightarrow(M, \omega)$ be as in Theorem 1.1. Let $Q_{N}: M \times M \rightarrow[0,+\infty)$ be the function given by

$$
Q_{N}(z, w)=\widetilde{G}\left(P_{N}(z, w)\right)=-\frac{1}{4 \pi^{2}} \int_{0}^{P_{N}(z, w)^{2}} \frac{\log (1-s)}{s} d s,
$$

where $P_{N}(z, w)$ is the normalized Szegö kernel given by (16). Then

$$
\operatorname{Var}\left(Z_{s^{N}}\right)=-\partial_{1} \bar{\partial}_{1} \partial_{2} \bar{\partial}_{2} Q_{N}
$$

Theorem 2.4 says that

$$
\operatorname{Var}\left(Z_{s^{N}}, \varphi\right)=\left(-\partial_{1} \bar{\partial}_{1} \partial_{2} \bar{\partial}_{2} Q_{N}, \varphi \otimes \varphi\right)=\int_{M \times M} Q_{N}(z, w) i \partial \bar{\partial} \varphi(z) \wedge i \partial \bar{\partial} \varphi(w)
$$

for all real $(m-1, m-1)$-forms $\varphi$ on $M$ with $\mathcal{C}^{2}$ coefficients.

Since $P_{N}^{2} \in \mathcal{C}^{\infty}(M \times M)$ and $P_{N}(z, w)<1$ for $z \neq w$, for sufficiently large $N$ (such that the Kodaira map for $L^{N}$ is an embedding), it follows from (20) that $Q_{N}$ is $\mathcal{C}^{\infty}$ off the diagonal, for $N \gg 0$.

Proposition 2.2 implies that the pluri-bipotential decays rapidly away from the diagonal:

Lemma 2.5. [SZ4, Lemma 3.4] For $b>\sqrt{j+q+1}, j \geq 0$, we have

$$
\left|\nabla^{j} Q_{N}(z, w)\right|=O\left(\frac{1}{N^{q}}\right), \quad \text { for } d(z, w)>\frac{b \sqrt{\log N}}{\sqrt{N}} .
$$

Proposition 2.3 yields the near-diagonal asymptotics:

Lemma 2.6. [SZ4, Lemma 3.5] For $b \in \mathbb{R}^{+}$, we have

$$
Q_{N}\left(z_{0}, z_{0}+\frac{v}{\sqrt{N}}\right)=\widetilde{G}\left(e^{-\frac{1}{2}|v|^{2}}\right)+O\left(N^{-1 / 2+\varepsilon}\right), \quad \text { for }|v| \leq b \sqrt{\log N}
$$


Recalling (21), we write,

$$
F(\lambda):=\widetilde{G}\left(e^{-\lambda}\right)=-\frac{1}{2 \pi^{2}} \int_{\lambda}^{\infty} \log \left(1-e^{-2 s}\right) d s \quad(\lambda \geq 0),
$$

so that $Q_{N}=F \circ\left(-\log P_{N}\right)$. By Proposition 2.3,

$$
-\log P_{N}\left(z_{0}, z_{0}+\frac{v}{\sqrt{N}}\right)=\frac{1}{2}|v|^{2}+O\left(|v|^{2} N^{-1 / 2+\varepsilon}\right) \text { for }|v|<b \sqrt{\log N} .
$$

It follows from Lemma 2.6 and (25) that $Q_{N} \in \mathcal{C}^{1}(M \times M)$ and the first partial derivatives of $Q_{N}$ vanish along the diagonal in $M \times M$, for $N \gg 0$. (We note that $Q_{N}$ is $\mathcal{C}^{\infty}$ off the diagonal, but is not $\mathcal{C}^{2}$ at all points on the diagonal in $M \times M$, as the computations in [SZ4] show.) We furthermore have the near-diagonal asymptotics:

Lemma 2.7. [SZ4, Lemma 3.7] There exist a constant $C_{m} \in \mathbb{R}^{+}$(depending only on the dimension $m$ ) and $N_{0}=N_{0}(M) \in \mathbb{Z}^{+}$such that for $N \geq N_{0}$, we have:

i) The coefficients of the current $\bar{\partial}_{1} \bar{\partial}_{2} Q_{N}$ are locally bounded functions (given by pointwise differentiation of $Q_{N}$ ), and we have the pointwise estimate

$$
\left|\bar{\partial}_{1} \bar{\partial}_{2} Q_{N}(z, w)\right| \leq C_{m} N \quad \text { for } 0<|w-z|<b \sqrt{\frac{\log N}{N}} .
$$

ii) If $m \geq 2$, the coefficients of the current $\partial_{1} \bar{\partial}_{1} \partial_{2} \bar{\partial}_{2} Q_{N}$ are locally $L^{m-1}$ functions, and we have the estimate

$$
\left|\partial_{1} \bar{\partial}_{1} \partial_{2} \bar{\partial}_{2} Q_{N}(z, w)\right| \leq \frac{C_{m} N}{|w-z|^{2}} \quad \text { for } \quad 0<|w-z|<b \sqrt{\frac{\log N}{N}} .
$$

Lemma 2.8. [SZ4, Lemma 3.9] For $N$ sufficiently large,

$$
\begin{array}{r}
-\partial_{1} \bar{\partial}_{1} \partial_{2} \bar{\partial}_{2} Q_{N}\left(z_{0}, z_{0}+\frac{v}{\sqrt{N}}\right)=N \operatorname{Var}_{\infty}^{z_{0}}(v)+O\left(|v|^{-2} N^{1 / 2+\varepsilon}\right), \\
\text { for } 0<|v|<b \sqrt{\log N},
\end{array}
$$

where $\operatorname{Var}_{\infty}^{z_{0}} \in T_{\left(z_{0}, v\right)}^{* 2,2}\left(M \times \mathbb{C}^{m}\right)$ is given by

$$
\begin{aligned}
\operatorname{Var}_{\infty}^{z_{0}}(v):= & -\frac{1}{16} F^{(4)}\left(\frac{1}{2}|v|^{2}\right) \partial(z \cdot \bar{v}) \wedge \bar{\partial}(\bar{z} \cdot v) \wedge \partial|v|^{2} \wedge \bar{\partial}|v|^{2} \\
& -\frac{1}{8} F^{(3)}\left(\frac{1}{2}|v|^{2}\right)\left[\partial \bar{\partial}|z|^{2} \wedge \partial|v|^{2} \wedge \bar{\partial}|v|^{2}+\bar{\partial}(\bar{z} \cdot v) \wedge \partial|v|^{2} \wedge \partial \bar{\partial}(z \cdot \bar{v})\right. \\
& \left.+\partial(z \cdot \bar{v}) \wedge \bar{\partial} \partial(\bar{z} \cdot v) \wedge \bar{\partial}|v|^{2}+\partial(z \cdot \bar{v}) \wedge \bar{\partial}(\bar{z} \cdot v) \wedge \partial \bar{\partial}|v|^{2}\right] \\
& -\frac{1}{4} F^{\prime \prime}\left(\frac{1}{2}|v|^{2}\right)\left[\bar{\partial} \partial(\bar{z} \cdot v) \wedge \partial \bar{\partial}(z \cdot \bar{v})+\partial \bar{\partial}|z|^{2} \wedge \partial \bar{\partial}|v|^{2}\right]
\end{aligned}
$$


Differentiating (24), we note that

$$
F^{\prime \prime}(\lambda)=\frac{1}{\pi^{2}} \frac{1}{e^{2 \lambda}-1}, \quad F^{(3)}(\lambda)=-\frac{1}{2 \pi^{2}} \operatorname{csch}^{2} \lambda, \quad F^{(4)}(\lambda)=\frac{1}{\pi^{2}} \operatorname{coth} \lambda \operatorname{csch}^{2} \lambda .
$$

Thus,

$$
F^{(j)}(\lambda)=(-1)^{j} \frac{(j-2) !}{2 \pi^{2}} \lambda^{-j+1}+O(1) \quad(\lambda>0),
$$

for $j \geq 2$, and hence

$$
\operatorname{Var}_{\infty}^{z_{0}}(v)=\left\{\begin{array}{lll}
O\left(|v|^{-2}\right) & \text { for } & |v|>0 \\
O\left(|v|^{4} e^{-|v|^{2}}\right) & \text { for } & |v|>1
\end{array}\right.
$$

3. The sharp variance estimate: Proof of Theorem 1.1

3.1. The codimension one case. To illustrate the basic ideas of the argument, we begin with the proof for the case $k=1$. By Theorem 2.4, we have

$$
\operatorname{Var}\left(Z_{s^{N}}, \varphi\right)=\int_{M} \mathcal{I}^{N}(z) i \partial \bar{\partial} \varphi(z)
$$

where

$$
\mathcal{I}^{N}(z)=\int_{\{z\} \times M} Q_{N}(z, w) i \partial \bar{\partial} \varphi(w)
$$

We let

denote the volume form of $M$, and we write

$$
\Omega_{M}=\frac{1}{m !} \omega^{m}
$$

$$
i \partial \bar{\partial} \varphi=\psi \Omega_{M}, \quad \psi \in \mathcal{C}_{\mathbb{R}}^{1}(M),
$$

so that

$$
\mathcal{I}^{N}(z)=\int_{\{z\} \times M} Q_{N}(z, w) \psi(w) \Omega_{M}(w) .
$$

To evaluate $\mathcal{I}^{N}\left(z_{0}\right)$ at a fixed point $z_{0} \in M$, we choose a normal coordinate chart centered at $z_{0}$ as in $\S 2$, and we make the change of variables $w=z_{0}+\frac{v}{\sqrt{N}}$. By Lemma 2.5 and $(32)-(33)$, we can approximate $\mathcal{I}^{N}\left(z_{0}\right)$ by integrating (33) over a small ball about $z_{0}$ :

$$
\begin{aligned}
\mathcal{I}^{N}\left(z_{0}\right)=\int_{|v| \leq b \sqrt{\log N}} Q_{N}\left(z_{0}, z_{0}+\frac{v}{\sqrt{N}}\right) \psi\left(z_{0}+\frac{v}{\sqrt{N}}\right) & \Omega_{M}\left(z_{0}+\frac{v}{\sqrt{N}}\right) \\
+ & O\left(\frac{1}{N^{2 m}}\right),
\end{aligned}
$$


where $b=\sqrt{m+2}$.

Since $\omega=\frac{i}{2} \partial \bar{\partial} \log a=\frac{i}{2} \partial \bar{\partial}\left[|z|^{2}+O\left(|z|^{3}\right)\right]$ in normal coordinates, we have

$\omega\left(z_{0}+\frac{v}{\sqrt{N}}\right)=\frac{i}{2} \sum\left[\delta_{j k}+O\left(\frac{|v|}{\sqrt{N}}\right)\right] \frac{1}{N} d v_{j} \wedge d \bar{v}_{k}=\frac{i}{2 N} \partial \bar{\partial}|v|^{2}+O\left(\frac{|v|}{N^{3 / 2}}\right)$,

for $|v| \leq b \sqrt{\log N}$. Hence

$\Omega_{M}\left(z_{0}+\frac{v}{\sqrt{N}}\right)=\frac{1}{m !}\left[\frac{i}{2 N} \partial \bar{\partial}|v|^{2}+O\left(\frac{|v|}{N^{3 / 2}}\right)\right]^{m}=\frac{1}{N^{m}}\left[\Omega_{E}(v)+O\left(\sqrt{\frac{\log N}{N}}\right)\right]$

for $|v| \leq b \sqrt{\log N}$, where

$$
\Omega_{E}(v)=\frac{1}{m !}\left(\frac{i}{2} \partial \bar{\partial}|v|^{2}\right)^{m}=\prod_{j=1}^{m} \frac{i}{2} d v_{j} \wedge d \bar{v}_{j}
$$

denotes the Euclidean volume form. Since $\varphi \in \mathcal{C}^{3}$ and hence $\psi\left(z+\frac{v}{\sqrt{N}}\right)=$ $\psi(z)+O(|v| / \sqrt{N})$, we then have by Lemma 2.6 and (34)-(36),

$$
\begin{aligned}
\mathcal{I}^{N}\left(z_{0}\right)=\frac{1}{N^{m}} & {\left[\int_{|v| \leq b \sqrt{\log N}}\left\{\widetilde{G}\left(e^{-\frac{1}{2}|v|^{2}}\right)+O\left(N^{-1 / 2+\varepsilon}\right)\right\}\left\{\psi\left(z_{0}\right)+O\left(N^{-1 / 2+\varepsilon}\right)\right\}\right.} \\
& \left.\times\left\{\Omega_{E}(v)+O\left(N^{-1 / 2+\varepsilon}\right)\right\}\right]+O\left(N^{-m-1}\right) \\
= & \frac{\psi\left(z_{0}\right)}{N^{m}}\left[\int_{|v| \leq b \sqrt{\log N}} \widetilde{G}\left(e^{-\frac{1}{2}|v|^{2}}\right) \Omega_{E}(v)+O\left(N^{-1 / 2+\varepsilon}\right)\right]
\end{aligned}
$$

Since $\widetilde{G}\left(e^{-\lambda}\right)=O\left(e^{-2 \lambda}\right)$ and hence

$$
\int_{|v| \geq b \sqrt{\log N}} \widetilde{G}\left(e^{-\frac{1}{2}|v|^{2}}\right) \Omega_{E}(v)=O\left(N^{-m-1}\right),
$$

we can replace the integral over the $(b \sqrt{\log N})$-ball with one over all of $\mathbb{C}^{m}$, and therefore

$$
\mathcal{I}^{N}\left(z_{0}\right)=\frac{\psi\left(z_{0}\right)}{N^{m}}\left[\int_{C^{m}} \widetilde{G}\left(e^{-\frac{1}{2}|v|^{2}}\right) \Omega_{E}(v)+O\left(N^{-1 / 2+\varepsilon}\right)\right]
$$


Recalling (20), we have

$$
\begin{aligned}
\int_{\mathbb{C}^{m}} \widetilde{G}\left(e^{-\frac{1}{2}|v|^{2}}\right) \Omega_{E}(v) & =\frac{1}{4 \pi^{2}} \sum_{k=1}^{\infty} \int_{\mathbb{C}^{m}} \frac{e^{-k|v|^{2}}}{k^{2}} \Omega_{E}(v) \\
& =\frac{1}{4 \pi^{2}} \sum_{k=1}^{\infty} \frac{\pi^{m}}{k^{m+2}}=\frac{\pi^{m-2}}{4} \zeta(m+2) .
\end{aligned}
$$

Therefore, by (30) and (39)-(40),

$$
\operatorname{Var}\left(Z_{s^{N}}, \varphi\right)=\frac{1}{N^{m}} \int_{M}\left[\frac{\pi^{m-2}}{4} \zeta(m+2)+O\left(N^{-1 / 2+\varepsilon^{\prime}}\right)\right] \psi(z)^{2} \Omega_{M}(z) .
$$

The variance formula of Theorem 1.1 for the case $k=1$ follows from (32) and (41).

3.2. An explicit formula for the variance. In this section, we give an integral formula for the variance of simultaneous zero currents in higher codimension (Corollary 3.3), which we shall use in the next section to derive the asymptotics of Theorem 1.1. This integral formula is a modification of the following formula for the variance:

TheOREm 3.1. [SZ4, Theorem 3.13] Let $1 \leq k \leq m$. Then for $N$ sufficiently large,

$$
\begin{aligned}
& \operatorname{Var}\left(Z_{s_{1}^{N}, \ldots, s_{k}^{N}}\right) \\
& =\partial_{1} \partial_{2}\left[\sum_{j=1}^{k}(-1)^{j-1}\left(\begin{array}{l}
k \\
j
\end{array}\right) \bar{\partial}_{1} \bar{\partial}_{2} Q_{N} \wedge\left(\partial_{1} \bar{\partial}_{1} \partial_{2} \bar{\partial}_{2} Q_{N}\right)^{j-1} \wedge\left(\mathbf{E} Z_{s^{N}} \otimes \mathbf{E} Z_{s^{N}}\right)^{k-j}\right] .
\end{aligned}
$$

where the current inside the brackets is an $L^{1}$ current on $M \times M$ given by pointwise multiplication, $Q_{N}$ is given by (22), and $\mathbf{E} Z_{s^{N}}$ is given by (13). Furthermore, $\operatorname{Var}\left(Z_{s_{1}^{N}, \ldots, s_{k}^{N}}\right)$ is an $L^{1}$ current on $M \times M$ if $k \leq m-1$.

By an $L^{1}$ current on the compact manifold $M \times M$, we mean a current whose local coefficients are $L^{1}$ functions. 
COROllary 3.2. Let $1 \leq k \leq m$. Then for $N$ sufficiently large,

$$
\begin{aligned}
& \operatorname{Var}\left(Z_{s_{1}^{N}, \ldots, s_{k}^{N}}\right) \\
& \quad=-\partial_{1} \bar{\partial}_{1} \partial_{2} \bar{\partial}_{2}\left[\sum_{j=1}^{k}\left(\begin{array}{c}
k \\
j
\end{array}\right) Q_{N}\left(-\partial_{1} \bar{\partial}_{1} \partial_{2} \bar{\partial}_{2} Q_{N}\right)^{j-1} \wedge\left(\mathbf{E} Z_{s^{N}} \otimes \mathbf{E} Z_{s^{N}}\right)^{k-j}\right] .
\end{aligned}
$$

where the current inside the brackets is an $L^{1}$ current on $M \times M$ given by pointwise multiplication.

Proof. Let

$$
T=\sum_{j=1}^{k}\left(\begin{array}{c}
k \\
j
\end{array}\right) Q_{N}\left(-\partial_{1} \bar{\partial}_{1} \partial_{2} \bar{\partial}_{2} Q_{N}\right)^{j-1} \wedge\left(\mathbf{E} Z_{s^{N}} \otimes \mathbf{E} Z_{s^{N}}\right)^{k-j}
$$

denote the expression inside the brackets in Corollary 3.2, regarded as a $(4 k-4)$ form on $M \times M \backslash \Delta$, where $\Delta=\{(z, z): z \in M\}$ denotes the diagonal. By Lemma 2.7(ii), $T=O\left(d(z, w)^{-2 k+2}\right)$ and hence $T$ defines an $L^{1}$ current on $M \times M$. It suffices to show that $\bar{\partial}_{1} \bar{\partial}_{2} T$ is an $L^{1}$ current and thus $\bar{\partial}_{1} \bar{\partial}_{2}$ can be moved outside the brackets in Theorem 3.1.

Let $U_{\varepsilon}=\{(z, w) \in M \times M: d(z, w)<\varepsilon\}$ denote the $\varepsilon$-neighborhood of the diagonal $\Delta$. For a test form $\varphi$ with $m-k+1 d z_{j}$ 's, $d \bar{z}_{j}$ 's, and $d w_{j}$ 's, and $m-k$ $d \bar{w}_{j}$ 's, we have

$$
\left(\bar{\partial}_{2} T, \varphi\right)=-\lim _{\varepsilon \rightarrow 0} \int_{M \times M \backslash U_{\varepsilon}} T \wedge \bar{\partial}_{2} \varphi=\lim _{\varepsilon \rightarrow 0} \int_{M \times M \backslash U_{\varepsilon}} \bar{\partial}_{2} T \wedge \varphi+\lim _{\varepsilon \rightarrow 0} \int_{\partial U_{\varepsilon}} T \wedge \varphi .
$$

Since $T \wedge \varphi=o\left(\varepsilon^{-2 m+1}\right)$ on $\partial U_{\varepsilon}$, the boundary integral goes to 0. By Lemma 2.7 (ii) and the fact that $Q_{N} \in \mathcal{C}^{1}(M \times M)$, the pointwise-defined form $\bar{\partial}_{2} T$ is also $O\left(d(z, w)^{-2 k+2}\right)$ and thus $\bar{\partial}_{2} T$ is an $L^{1}$ current on $M \times M$ given by pointwise differentiation.

Repeating the same argument with $T$ replaced by $\bar{\partial}_{2} T$ and using part (i) of Lemma 2.7 as well as part (ii), we then conclude that $\bar{\partial}_{1} \bar{\partial}_{2} T$ is an $L^{1}$ current.

Corollary 3.2 can also be shown directly, using the argument in the proof of Theorem 3.1 in [SZ4]. 
COROLlary 3.3. The variance in Theorem 1.1 is given by:

$$
\begin{aligned}
& \operatorname{Var}\left(\left[Z_{s_{1}^{N}, \ldots, s_{k}^{N}}\right], \varphi\right) \\
= & \sum_{j=1}^{k}\left(\begin{array}{c}
k \\
j
\end{array}\right) \int_{M \times M} Q_{N}\left(-\partial_{1} \bar{\partial}_{1} \partial_{2} \bar{\partial}_{2} Q_{N}\right)^{j-1} \wedge\left(\mathbf{E} Z_{s^{N}} \bigotimes \mathbf{E} Z_{s^{N}}\right)^{k-j} \wedge(i \partial \bar{\partial} \varphi \bigotimes i \partial \bar{\partial} \varphi),
\end{aligned}
$$

where the integrands are in $L^{1}(M \times M)$.

3.3. Higher codimensions. Recalling (14), we write the formula of Corollary 3.3 as follows:

$$
\begin{aligned}
\operatorname{Var}\left(\left[Z_{s_{1}^{N}, \ldots, s_{k}^{N}}\right], \varphi\right) & =\sum_{j=1}^{k}\left(\begin{array}{c}
k \\
j
\end{array}\right) V_{j}^{N}(\varphi) \\
V_{j}^{N}(\varphi) & =\left(\frac{N}{\pi}\right)^{2 k-2 j} \int_{M \times M} Q_{N}(z, w)\left[-\partial_{1} \bar{\partial}_{1} \partial_{2} \bar{\partial}_{2} Q_{N}(z, w)\right]^{j-1} \\
\wedge\left[\omega(z)^{k-j}+O\left(\frac{1}{N}\right)\right] & \wedge\left[\omega(w)^{k-j}+O\left(\frac{1}{N}\right)\right] \wedge i \partial \bar{\partial} \varphi(z) \wedge i \partial \bar{\partial} \varphi(w) \\
& =\left(\frac{N}{\pi}\right)^{2 k-2 j} \int_{M} \mathcal{I}_{j}^{N} \wedge\left[\omega^{k-j} \wedge i \partial \bar{\partial} \varphi+O\left(\frac{1}{N}\right)\right]
\end{aligned}
$$

where

$$
\begin{aligned}
\mathcal{I}_{j}^{N}(z)=\int_{\{z\} \times M} & Q_{N}(z, w)\left[-\partial_{1} \bar{\partial}_{1} \partial_{2} \bar{\partial}_{2} Q_{N}(z, w)\right]^{j-1} \\
& \wedge\left[\omega(w)^{k-j} \wedge i \partial \bar{\partial} \varphi(w)+O\left(\frac{1}{N}\right)\right] \in T_{z}^{* j-1, j-1}(M) .
\end{aligned}
$$

The integrand in (44) is regarded as an $(m, m)$-form (in the $w$ variable) with values in $T_{z}^{* j-1, j-1}(M)$.

Fix a point $z_{0} \in M$, and let $1 \leq j \leq k \leq m$. To evaluate $\mathcal{I}_{j}^{N}\left(z_{0}\right)$, we write

$$
\begin{aligned}
\omega^{k-j} \wedge i \partial \bar{\partial} \varphi & =\sum \psi_{J K}(w) d w^{J} \wedge d \bar{w}^{K} \\
& =\frac{1}{N^{m-j+1}} \sum \psi_{J K}\left(z_{0}+\frac{v}{\sqrt{N}}\right) d v^{J} \wedge d \bar{v}^{K}, \quad|J|=|K|=m-j+1
\end{aligned}
$$


By Lemma 2.5, we can replace integration over $M$ in (44) with integration over the small ball of radius $b \sqrt{\log N / N}$, with $b=\sqrt{m+4}$, to obtain:

$$
\begin{aligned}
& \mathcal{I}_{j}^{N}\left(z_{0}\right) \\
=N^{-m+j-1} & \int_{|v| \leq b \sqrt{\log N}} Q_{N}\left(z_{0}, z_{0}+\frac{v}{\sqrt{N}}\right)\left[-\partial_{1} \bar{\partial}_{1} \partial_{2} \bar{\partial}_{2} Q_{N}\left(z_{0}, z_{0}+\frac{v}{\sqrt{N}}\right)\right]^{j-1} \\
& \wedge \sum\left[\psi_{J K}\left(z_{0}+\frac{v}{\sqrt{N}}\right)+O\left(\frac{1}{N}\right)\right] d v^{J} \wedge d \bar{v}^{K}+O\left(N^{-m-1}\right) .
\end{aligned}
$$

By Lemma 2.7, the above integrand is $L^{1}$, and hence by Lemma 2.8 ,

$$
\begin{aligned}
& \mathcal{I}_{j}^{N}\left(z_{0}\right)=N^{2 j-2-m}\left[\int_{|v| \leq b \sqrt{\log N}} F\left(\frac{1}{2}|v|^{2}\right)\left\{\operatorname{Var}_{\infty}^{z_{0}}(v)\right\}^{j-1} \sum \psi_{J K}\left(z_{0}\right) d v^{J} \wedge d \bar{v}^{K}\right. \\
& \left.+O\left(N^{-\frac{1}{2}+\varepsilon}\right)\right] \\
& =N^{2 j-2-m}\left[\sum \psi_{J K}\left(z_{0}\right) \int_{v \in \mathbb{C}^{m}} F\left(\frac{1}{2}|v|^{2}\right)\left\{\operatorname{Var}_{\infty}^{z_{0}}(v)\right\}^{j-1} d v^{J} \wedge d \bar{v}^{K}\right. \\
& \left.+O\left(N^{-\frac{1}{2}+\varepsilon}\right)\right] .
\end{aligned}
$$

Here, we replaced the integral over the $(b \sqrt{\log N})$-ball with one over all of $\mathbb{C}^{m}$, since by (29) we have $F\left(\frac{1}{2}|v|^{2}\right)\left\{\operatorname{Var}_{\infty}^{z_{0}}(v)\right\}^{j-1}=O\left(e^{-|v|^{2}}\right)$ for $|v|>1$, and hence

$$
\int_{|v|>b \sqrt{\log N}} F\left(\frac{1}{2}|v|^{2}\right)\left\{\operatorname{Var}_{\infty}^{z_{0}}(v)\right\}^{j-1} d v^{J} \wedge d \bar{v}^{K}=O\left(N^{-4 m}\right) .
$$

It follows from (43) and (45) that

$$
V_{j}^{N}(\varphi)=N^{2 k-m-2}\left[\int_{M} \sum B_{J K A B}^{j} \psi_{J K} \bar{\psi}_{A B} \Omega_{M}+O\left(N^{-\frac{1}{2}+\varepsilon}\right)\right],
$$

where $B^{j}=\left\{B_{J K A B}^{j}\right\}$ is a universal Hermitian form on $T^{* m-j+1, m-j+1}(M)$. Theorem 1.1 then follows from (42) and (46) with

$$
B_{m k}(\alpha, \alpha)=\sum_{j=1}^{k} B^{j}\left(\omega^{k-j} \wedge \alpha, \omega^{k-j} \wedge \alpha\right) .
$$




\section{Asymptotic normality: Proof of Theorem 1.2}

The proof of Theorem 1.2 is an application of Propositions 2.2-2.3 to a general result of Sodin-Tsirelson [ST] on a central limit theorem, or asymptotic normality, for nonlinear functionals of Gaussian processes. Following [ST], we define a normalized complex Gaussian process to be a complex-valued random function $w(t)$ on a measure space $(T, \mu)$ of the form

$$
w(t)=\sum c_{j} g_{j}(t)
$$

where the $c_{j}$ are i.i.d. complex Gaussian random variables (of mean 0, variance 1 ), and the $g_{j}$ are (fixed) complex-valued measurable functions such that

$$
\sum\left|g_{j}(t)\right|^{2}=1 \quad \text { for all } t \in T \text {. }
$$

We let $w^{1}, w^{2}, w^{3}, \ldots$ be a sequence of normalized complex Gaussian processes on a finite measure space $(T, \mu)$. Let $f(r) \in L^{2}\left(\mathbb{R}^{+}, e^{-r^{2} / 2} r d r\right)$ and let $\psi: T \rightarrow \mathbb{R}$ be bounded measurable. We write

$$
Z_{N}^{\psi}\left(w^{N}\right)=\int_{T} f\left(\left|w^{N}(t)\right|\right) \psi(t) d \mu(t)
$$

TheOREM 4.1. [ST, Theorem 2.2] Let $\rho_{N}(s, t)$ be the covariance functions for the Gaussian processes $w^{N}(t)$. Suppose that

i) $\quad \liminf _{N \rightarrow \infty} \frac{\int_{T} \int_{T}\left|\rho_{N}(s, t)\right|^{2 \alpha} \psi(s) \psi(t) d \mu(s) d \mu(t)}{\sup _{s \in T} \int_{T}\left|\rho_{N}(s, t)\right| d \mu(t)}>0$, for $\alpha=1$ if $f$ is monotonically increasing, or for all $\alpha \in \mathbb{Z}^{+}$otherwise;

ii) $\quad \lim _{N \rightarrow \infty} \sup _{s \in T} \int_{T}\left|\rho_{N}(s, t)\right| d \mu(t)=0$.

Then the distributions of the random variables

$$
\frac{Z_{N}^{\psi}-\mathbf{E} Z_{N}^{\psi}}{\sqrt{\operatorname{Var}\left(Z_{N}^{\psi}\right)}}
$$

converge weakly to $\mathcal{N}(0,1)$ as $N \rightarrow \infty$.

We apply this result with $f(r)=\log r$ and $(T, \mu)=\left(M, \Omega_{M}\right)$. To define our normalized Gaussian processes $w^{N}$ on $M$, we choose a measurable section $\sigma_{L}: M \rightarrow L$ of $L$ with $\left\|\sigma_{L}(z)\right\|_{h}=1$ for all $z \in M$, and we let

$$
S_{j}^{N}=F_{j}^{N} \sigma_{L}^{\otimes N}, \quad j=1, \ldots, d_{N},
$$


be an orthonormal basis for $H^{0}\left(M, L^{N}\right)$ with respect to its Hermitian Gaussian measure, for each $N \in \mathbb{Z}^{+}$. We then let

$$
g_{j}^{N}(z):=\frac{F_{j}^{N}(z)}{\sqrt{\Pi_{N}(z, z)}}, \quad j=1, \ldots, d_{N} .
$$

Since $\left|F_{j}^{N}\right|=\left\|S_{j}^{N}\right\|_{h^{N}}$, it follows that $w^{N}=\sum c_{j} g_{j}^{N}$ defines a normalized complex Gaussian process, for each $N \in \mathbb{Z}^{+}$(where the $c_{j}$ are i.i.d. standard complex Gaussian random variables). In fact,

$$
\left|w^{N}(z)\right|=\frac{\left\|s^{N}(z)\right\|_{h^{N}}}{\sqrt{\Pi_{N}(z, z)}},
$$

where

$$
s^{N}=\sqrt{\Pi_{N}(z, z)} w^{N} \sigma_{L}^{\otimes N}=\sum c_{j} S_{j}^{N}
$$

is a random holomorphic section in $H^{0}\left(M, L^{N}\right)$. The covariance functions $\rho_{N}(z, w)$ for these Gaussian processes satisfy

$$
\left|\rho_{N}(z, w)\right|=P_{N}(z, w) .
$$

We now let $\varphi$ be a fixed $\mathcal{C}^{3}$ real $(m-1, m-1)$-form on $M$ and we write

$$
\frac{i}{\pi} \partial \bar{\partial} \varphi=\psi \Omega_{M} .
$$

Then $\psi \in \mathcal{C}^{1}$, and

$$
Z_{N}^{\psi}\left(w^{N}\right)=\int_{M}\left(\log \left\|s^{N}(z)\right\|_{h^{N}}-\log \sqrt{\Pi_{N}(z, z)}\right) \frac{i}{\pi} \partial \bar{\partial} \varphi(z)=\left(Z_{s^{N}}, \varphi\right)+k_{N},
$$

where the $k_{N}$ are constants (depending on $L \rightarrow M$ and $\varphi$, but independent of the random sections $\left.s^{N}\right)$. Hence $Z_{N}^{\psi}\left(w^{N}\right)$ has the same variance as the smooth linear statistic $\left(Z_{s^{N}}, \varphi\right)$, and it suffices by Theorem 4.1 to check that the covariance function satisfies conditions (i)-(ii) of the theorem. We start with (ii): by Proposition 2.2,

$$
\lim _{N \rightarrow \infty} \sup _{z \in M} \int_{d(z, w)>b \sqrt{\frac{\log N}{N}}} P_{N}(z, w) \Omega_{M}(w)=0 .
$$

On the other hand, since $P_{N}(z, w) \leq 1$, it is obvious that the same limit holds for $d(z, w) \leq b \sqrt{\frac{\log N}{N}}$, verifying (ii).

To check (i), we again break up the integral into the near diagonal $d(z, w) \leq$ $b \sqrt{\frac{\log N}{N}}$ and the off-diagonal $d(z, w)>b \sqrt{\frac{\log N}{N}}$. As before, the integrals over the 
off-diagonal set tend to zero rapidly and can be ignored in both the numerator and denominator.

On the near diagonal, we replace $P_{N}$ by its asymptotics in Proposition 2.3. The asymptotic formula for $P_{N}$ has a universal leading term independent of $z$ and has uniform remainder, so condition (i) (with $\alpha=1$ ) becomes

$$
\liminf _{N \rightarrow \infty} \frac{\int_{M} \Omega_{M}(z) \int_{|u|<b \sqrt{\log N} e^{-|u|^{2}}\left[1+R_{N}(u)\right]^{2} \psi\left(z+\frac{u}{\sqrt{N}}\right) \psi(z) d u}}{\int_{|u|<b \sqrt{\log N}} e^{-\frac{1}{2}|u|^{2}}\left[1+R_{N}(u)\right] d u}>0 .
$$

Since $\psi \in \mathcal{C}^{1}$, the ratio clearly tends to $2^{-m} \int_{M} \psi(z)^{2} \Omega_{M}>0$, which verifies (i) and completes the proof of Theorem 1.2.

\section{Open PRoblems on SMOOTh AND COUNTing Statistics}

In this section, we present a number of open problems on smooth and discontinuous linear statistics of zeros.

(1) Asymptotic normality of the smooth linear statistics $\left(Z_{s_{1}^{N}, \ldots, s_{k}^{N}}, \varphi\right)$ has only been proved in codimension one, i.e. when $k=1$. But these random variables are likely to be asymptotically normal for all dimensions $m$ and codimensions $k$. It would be interesting to prove (or disprove) this statement, in particular for $k=m$.

(2) To our knowledge, no results are known to date regarding the asymptotic normality of the counting statistics $\mathcal{N}_{N}^{U}\left(s_{1}^{N}, \ldots, s_{m}^{N}\right)$. This is analogous to, but presumably harder than, the smooth linear statistic when $k=m$.

(3) In [Zh], Qi Zhong obtained surprising results on the expected value of the 'energy' random variable

$$
\mathcal{E}_{G}\left(s^{N}\right)=\sum_{i \neq j} G\left(a_{i}, a_{j}\right), \quad Z_{s^{N}}=\left\{a_{1}, \ldots, a_{N}\right\},
$$

summing the values of the Green's function $G$ over pairs of distinct zeros of a random polynomial or section $s^{N} \in H^{0}\left(M, L^{N}\right)$ of the $N$-th power of a positive line bundle $(L, h)$ over a compact Riemann surface $M$. (The Green's function is normalized to equal $+\infty$ on the diagonal.) Zhong proved that when $G$ is the Green's function for the Riemannian metric induced by the curvature of $h$, the expected energy has the asymptotics $\mathbf{E} \mathcal{E}_{G}\left(p_{N}\right) \sim-\frac{1}{4 \pi} N \log N$. It is known (N. Elkies) that $-\frac{1}{4 \pi} N \log N$ is 
also the asymptotic minimum for the energy sum (47). The energy is a partially smooth linear statistic

$$
\left(G, Z_{s^{N}} \otimes Z_{s^{N}}-\Delta_{Z_{s^{N}}}\right)
$$

on $M \times M$, where $\Delta_{Z_{s^{N}}}$ represents the diagonal terms of $Z_{s^{N}} \otimes Z_{s^{N}}$. The statistic (48) is not smooth since $G$ has a logarithmic singularity and since we subtracted the diagonal current. It is a random variable of one section $s_{N}$ in dimension one, but $G$ is a function on $M \times M$, so the variance of $\mathcal{E}_{G}$ involves the rather complicated four-point correlation function of $Z_{s}$ rather than the pair correlation, for which Theorem 2.4 gives a useful formula. It would be interesting to investigate the variance of the energy $\mathcal{E}_{G}$. It seems that it should tend to zero with $N$ since the Gaussian measure is concentrated on 'polynomials' whose zeros are asymptotic minimizers.

(4) The expected distribution of zeros can have quite disparate asymptotics when the ensembles are given Gaussian measures induced from inner products on the space of polynomials (or sections) which use non-smooth volume forms or non-positively curved line bundle metrics. For instance, in the case where the measure is supported on an analytic plane domain $\Omega \subset \mathbb{C}$ or on its boundary, it was shown in [SZ3] that the expected distributions of random zeros of random polynomials of degree $N$ tend to the equilibrium measure of $\Omega$. This result was generalized to higher dimensions and more general metrics and measures in [B11, BS, Be1, Be2], where it is shown that the expected distribution of zeros tends to an equilibrium measure adapted to the measure and metric. In [Sh], an upper bound was given for the variances of the smooth linear statistics when the inner products are defined by arbitrary measures (and also for more general sequences of ensembles of increasing degrees). This bound is sufficient to prove that sequences of random zeros in these ensembles almost surely converge to their equilibrium measure, although the bound is not always sharp. The Szegö kernels for the inner products in [SZ3, Bl1, BS, Be1, Be2] are quite different from Szegő kernels for positive line bundles in this article, and so the asymptotics of the variances might be quite different. It would be interesting to determine them.

(5) Results on expected values for the analogous $\mathrm{SO}(2)$ and $\mathrm{SO}(m+1)$ ensembles of random real polynomials in one or several variables were given by 
[BD, EK, Ro, SS], and a (global) variance result for real zeros was given in $[\mathrm{Ws}]$. Maslova $[\mathrm{Ma}]$ proved the asymptotic normality of the number of real zeros for the Kac ensemble [Kac] of random real polynomials on $\mathbb{R}^{1}$ (as well as for some non-Gaussian ensembles). But as far as we are aware, asymptotic normality for numbers of real zeros in the $\mathrm{SO}(m+1)$ ensemble has not been investigated.

\section{REFERENCES}

[Be1] R. Berman, Bergman kernels and equilibrium measures for line bundles over projective manifolds, Amer. J. Math. 131 (2009), 1485-1524.

[Be2] R. Berman, Bergman kernels for weighted polynomials and weighted equilibrium measures of $\mathbb{C}^{n}$, Indiana Univ. Math. J. 58 (2009), 1921-1946.

[BD] P. Bleher and X. Di, Correlations between zeros of a random polynomial, J. Statist. Phys. 88 (1997), 269-305.

[BSZ1] P. Bleher, B. Shiffman and S. Zelditch, Poincaré-Lelong approach to universality and scaling of correlations between zeros, Comm. Math. Phys. 208 (2000), 771-785.

[BSZ2] P. Bleher, B. Shiffman and S. Zelditch, Universality and scaling of correlations between zeros on complex manifolds, Invent. Math. 142 (2000), 351-395.

[Bl1] T. Bloom, Random polynomials and Green functions, Int. Math. Res. Not. 2005 (2005), 1689-1708.

[BS] T. Bloom and B. Shiffman, Zeros of random polynomials on $\mathbb{C}^{m}$, Math. Res. Lett. 14 (2007), 469-479.

[BBL] E. Bogomolny, O. Bohigas, and P. Leboeuf, Quantum chaotic dynamics and random polynomials, J. Statist. Phys. 85 (1996), 639-679.

[Ca] D. Catlin, The Bergman kernel and a theorem of Tian, in: Analysis and Geometry in Several Complex Variables, G. Komatsu and M. Kuranishi, eds., Birkhäuser, Boston, 1999.

[EK] A. Edelman and E. Kostlan, How many zeros of a random polynomial are real? Bull. Amer. Math. Soc. 32 (1995), 1-37.

[FH] P. J. Forrester and G. Honner, Exact statistical properties of the zeros of complex random polynomials, J. Phys. A 32 (1999), 2961-2981.

[Ha] J. H. Hannay, Chaotic analytic zero points: exact statistics for those of a random spin state, J. Phys. A 29 (1996), L101-L105.

[Kac] M. Kac, On the average number of real roots of a random algebraic equation, II, Proc. London Math. Soc. 50 (1949), 390-408.

[Ma] N. B. Maslova, The distribution of the number of real roots of random polynomials (Russian) Teor. Verojatnost. i Primenen. 19 (1974), 488-500 (English translation: Theor. Probability Appl. 19 (1974), 461-473).

[NV] S. Nonnenmacher and A. Voros, Chaotic eigenfunctions in phase space, J. Statist. Phys. 92 (1998), 431-518. 
[Ro] J. M. Rojas, On the average number of real roots of certain random sparse polynomial systems, The mathematics of numerical analysis (Park City, UT, 1995), 689-699, Lectures in Appl. Math. 32, Amer. Math. Soc., Providence, RI, 1996.

[Sh] B. Shiffman, Convergence of random zeros on complex manifolds, Special issue for Qikeng Lu, Sci. China Ser. A 51 (2008), 707-720.

[SZ1] B. Shiffman and S. Zelditch, Distribution of zeros of random and quantum chaotic sections of positive line bundles, Comm. Math. Phys. 200 (1999), 661-683.

[SZ2] B. Shiffman and S. Zelditch, Asymptotics of almost holomorphic sections of ample line bundles on symplectic manifolds, J. Reine Angew. Math. 544 (2002), 181-222.

[SZ3] B. Shiffman and S. Zelditch, Equilibrium distribution of zeros of random polynomials, Int. Math. Res. Not. 2003 (2003), 25-49.

[SZ4] B. Shiffman and S. Zelditch, Number variance of random zeros on complex manifolds, Geom. Funct. Anal., 18 (2008) 1422-1475.

[SZZ] B. Shiffman, S. Zelditch and S. Zrebiec, Overcrowding and hole probabilities for random zeros on complex manifolds, Indiana Univ. Math. J. 57 (2008), 1977-1997.

[SS] M. Shub and S. Smale, Complexity of Bezout's theorem. II. Volumes and probabilities, Computational algebraic geometry (Nice, 1992), 267-285, Progr. Math. 109, Birkhäuser Boston, Boston, MA, 1993.

[ST] M. Sodin and B. Tsirelson, Random complex zeros, I. Asymptotic normality, Israel J. Math. 144 (2004), 125-149.

[Ti] G. Tian, On a set of polarized Kähler metrics on algebraic manifolds, J. Diff. Geometry 32 (1990), 99-130.

[Ws] M. Wschebor, On the Kostlan-Shub-Smale model for random polynomial systems. Variance of the number of roots, J. Complexity 21 (2005), 773-789.

[Ze] S. Zelditch, Szegö kernels and a theorem of Tian, Internat. Math. Res. Notices 1998 (1998), 317-331.

[Zh] Q. Zhong, Energy of zeros of random sections on Riemann surfaces, Indiana Univ. Math. J. 57 (2008), 1753-1780.

Bernard Shiffman

Department of Mathematics, Johns Hopkins University,

Baltimore, MD 21218, USA

E-mail: shiffman@math.jhu.edu

Steve Zelditch

Department of Mathematics, Johns Hopkins University,

Baltimore, MD 21218, USA

E-mail: zelditch@math.jhu.edu 